\title{
Reativações Rúpteis de Zonas de Cisalhamento Durante o Cretáceo-Quaternário nas Bacias Sergipe-Alagoas e Pernambuco
}

\author{
David L. Vasconcelos ${ }^{1}$, Francisco H. R. Bezerra ${ }^{1}$, Walter E. Medeiros ${ }^{1,2}$, David L. de Castro ${ }^{1}$, Gilsijane V. Ramos ${ }^{1}$ \\ 1 - Programa de Pós-Graduação em Geodinâmica e Geofísica - UFRN \\ 2 - Instituto Nacional de Geofísica do Petróleo - INCT-GP (CNPq)
}

Copyright 2016, SBGf - Sociedade Brasileira de Geofísica

Este texto foi preparado para a apresentação no VII Simpósio Brasileiro de Geofísica, Ouro Preto, 25 a 27 de outubro de 2016. Seu conteúdo foi revisado pelo Comitê Técnico do VII SimBGf, mas não necessariamente representa a opinião da SBGf ou de seus associados. É proibida a reprodução total ou parcial deste material para propósitos comerciais sem prévia autorização da SBGf.

\section{Resumo}

Essa pesquisa integra dados aeromagnéticos e de sensoriamento remoto com uma compilação de dados geológicos para identificar reativações rúpteis de zonas de cisalhamento do embasamento na margem continental do Nordeste brasileiro. A interpretação dos dados aeromagnéticos permitiu a identificação de continuidades das zonas de cisalhamento na região das bacias marginais. No embasamento, os lineamentos magnéticos representam zonas de cisalhamento dúcteis Pré-cambrianas. No entanto, nas bacias estes traços representam falhas reativadas das estruturas do embasamento. Nós concluímos que essas reativações ocorreram principalmente no Cretáceo, durante a abertura do Atlântico Sul. Entretanto, nós observamos que tanto as falhas do Cretáceo quanto as zonas de cisalhamento Pré-cambrianas foram reativadas durante 0 Cenozoico. Essas reativações têm implicações para a evolução das bacias.

\section{Introdução}

Reativações rúpteis de zonas de cisalhamento do embasamento têm sido registradas em várias margens continentais, tais como no nordeste da Antártida (Storti et al., 2007), no sul do Vietnã (Fyhn et al., 2009) e no noroeste da Namíbia (Salomon et al., 2015). A reativação dessas estruturas do embasamento tem sido apontada como um dos principais fatores na evolução de margens continentais. Portanto, assimilar os fatores controladores e mecanismos envolvidos nos padrões de estruturas rúpteis em margens continentais é fundamental para entender a evolução de margens de uma maneira geral (Eig e Bergh, 2011).

Várias bacias marginais da América do Sul possuem influências de reativações rúpteis de zonas de cisalhamento (p. ex., Bezerra et al., 2014; Lima et al., 2016). Muitas dessas reativações possuem ligação com a abertura do Atlântico Sul (Matos, 1992), iniciada durante o Triássico-Jurássico (225-160 Ma) e com ápice no Jurássico-Cretáceo (160-115 Ma), as quais influenciaram na evolução tectono-estratigráfica destas bacias (Bezerra et al., 2011).

Contudo, essas reativações não se restringem a fase rifte. Reativações tectônicas de estruturas preexistentes na fase pós-rifte são registradas em várias margens continentais. Por exemplo, no sudeste da China (Mao et al., 2015), no sudoeste de Portugal (Zitellini et al., 2004) e no nordeste da Guiana Francesa (Basile et al., 2013).

Na margem continental brasileira, ao longo do CretáceoCenozoico, foram descritas reativações pós-rifte de zonas de cisalhamento e de falhas geradas durante a abertura do Atlântico Sul (Bezerra et al., 2014; Lima et al., 2014). Essas reativações tem ocasionado deformações nas porções offshore (Barbosa et al., 2014) e onshore de bacias da margem continental (Bezerra et al., 2014). Inclusive, essas deformações influenciaram na deposição dos sedimentos (Lima et al., 2014) e no controle das formas de relevo (Lima et al., 2016) destas bacias.

Apesar de algumas pesquisas terem mostrado a reativação de zonas de cisalhamento em bacias da margem continental brasileira (Bezerra et al., 2014; Lima et al., 2016; Matos, 1992), pouco se sabe a respeito da influência destas reativações na deposição dos sedimentos e na compartimentação estrutural nas fase rifte e pós-rifte das bacias Sergipe-Alagoas e Pernambuco. Nesta pesquisa nós investigamos a influência de reativações rúpteis de zonas de cisalhamento dúcteis do embasamento cristalino na deposição e deformação das unidades sedimentares na fase rifte e pós-rifte das bacias Sergipe-Alagoas e Pernambuco (Figura 1A).

Neste contexto, a compreensão das divisões tectônicas e litoestratigrafia da Província Borborema é fundamental para entender a influência de reativações de zonas de cisalhamento na deposição e deformação das unidades sedimentares nas bacias marginais do Nordeste do Brasil. A Província Borborema (Figura 1) é um mosaico de porções de embasamento e faixas orogênicas de idades arqueanas a neoproterozoicas separadas por zonas de cisalhamento (Brito Neves et al., 2014). O último grande evento tectônico que afetou esta província ocorreu no Mesozoico, durante a fragmentação do Gondwana e teve como resultado o desenvolvimento das bacias marginais, tais como as bacias Sergipe-Alagoas e Pernambuco (Matos, 1992).

A Bacia Sergipe-Alagoas (Figura 1B) possui um dos mais extensos registros sedimentares da margem continental da América do Sul, apresentando unidades dos estágios sinéclise, pré-rifte, rifte, transicional e pós-riftes (Matos, 1992; Souza-Lima et al., 2002). Por outro lado, a Bacia Pernambuco (Figura 1B), considerada como um dos últimos elos entre a América do Sul e a África (Matos, 1992), apresenta apenas registros sedimentares dos estágios rifte e pós-rifte (Lima Filho, 1998). Ambas registraram reativações de estruturas rúpteis, as quais ocorreram desde o Cretáceo até o Quaternário (Almeida et al., 2005; Lima et al., 2014). 


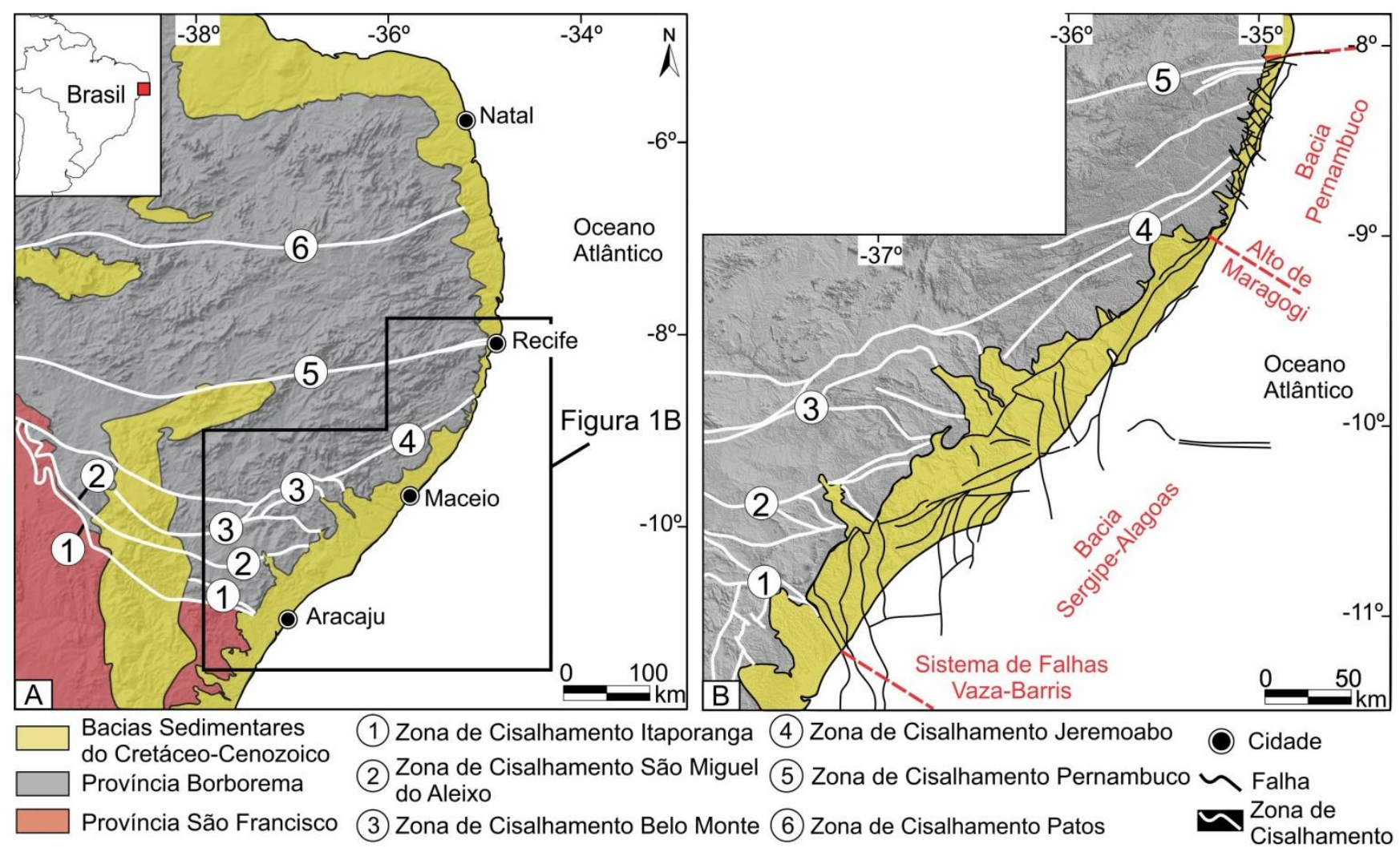

Figura 1 - (A) Mapa da Margem Continental de parte do Nordeste brasileiro com as principais zonas de cisalhamento da área de estudo. (B) Mapa de falhas compiladas de trabalhos citados no texto das bacias Sergipe-Alagoas e Pernambuco.

\section{Metodologia}

Inicialmente, nós confeccionamos um mapa estrutural da área de pesquisa (Figura 1B) através da compilação de mapas preexistentes, com foco nas principais estruturas do embasamento cristalino e das bacias marginais (Almeida et al., 2005; Angelim e Wanderley, 2004; Angelim et al., 2004a; Barbosa et al., 2014; Bezerra et al., 2014; D'el-Rey Silva, 1999; Delgado et al., 2003; Falkenhein et al., 1986; Kosin et al., 2004; Lima Filho, 1998; Souza-Lima et al., 2002).

Posteriormente, nós utilizamos dados dos projetos aerogeofísicos Estado de Sergipe (LASA PROSPRECÇÕES S/A, 2011), Paulo Afonso-Teotônio Vilela (Microsurvey Aerogeofísica e Consultoria Científica Ltda., 2011) e Borda Leste do Planalto da Borborema (LASA PROSPRECÇÕES S/A, 2008), cedidos pelo Serviço Geológico do Brasil (CPRM). Estes projetos levantaram perfis magnéticos, com linhas de voo e controle espaçadas de 500 e 10.000 m, orientadas nas direções N-S e E-W, respectivamente, e com altura de voo fixada em $100 \mathrm{~m}$ sobre o terreno. Estes levantamentos realizaram o magnetômetro com sensor de vapor de césio, montado na cauda da aeronave (tipo stinger), sendo as medidas efetuadas a cada 0,1 segundo. Os dados fornecidos pela CPRM já estavam micronivelados e corrigidos do IGRF (International Geomagnetic Reference Field). Estes projetos seguiram, aproximadamente, a da altura de voo fixada em 100m em relação à superfície do terreno, com exceção das áreas referentes às regiões metropolitanas de Aracaju, Maceió e Recife.

Os dados aeromagnéticos foram processados através do software Oasis Montaj 8.2 da Geosoft. Os mesmos foram interpolados utilizando o método Bidirecional, gerando um mapa do Campo Magnético Anômalo (CMA) com célula de $250 \mathrm{~m}$. Posteriormente foi gerado o mapa da Inclinação do Sinal Analítico (ISA - Figura 2), onde foram interpretados os lineamentos magnéticos. Para correlação das anomalias magnéticas com feições geomorfológicas, nós utilizamos imagens da Shuttle Radar Topography Mission (SRTM) refinada pelo projeto TOPODATA (Valeriano e Albuquerque, 2010) do Instituto Nacional de Pesquisas Espaciais $(30 \mathrm{~m}$ de resolução espacial). Nós processamos estas imagens com filtros de iluminação Hillshade (45ㅇ e $\left.315^{\circ}\right)$ para realçar as estruturas com direções NW-SE e NE-SW, respectivamente. Por fim, nós sobrepomos a estas imagens os lineamentos magnéticos interpretados para observarmos a influência de zonas cisalhamento no relevo.

\section{Resultados}

As investigações focaram no reconhecimento de zonas de cisalhamento do embasamento das bacias SergipeAlagoas e Pernambuco. Inicialmente foram analisadas as continuidades das principais estruturas geológicas na porção das bacias marginais. Para isso, utilizamos a 
compilação dessas estruturas (Figura 1B) correlacionando-as com as anomalias magnéticas observadas no mapa ISA (Figura 2).

Algumas zonas de cisalhamento mapeadas (Figura 2), tais como Itaporanga $(\mathrm{ZCl})$, São Miguel do Aleixo (ZCSMA), Belo Monte (ZCBM), Jeremoabo (ZCJ) e Pernambuco (ZCPE), coincidem com os lineamentos magnéticos interpretados. Essas anomalias magnéticas observadas através do mapa ISA foram interpretadas como estruturas do embasamento cristalino. A continuidade dessas feições estruturais para a porção das bacias sedimentares da margem continental foi correlacionada com as falhas mapeadas nas bacias por trabalhos anteriores.

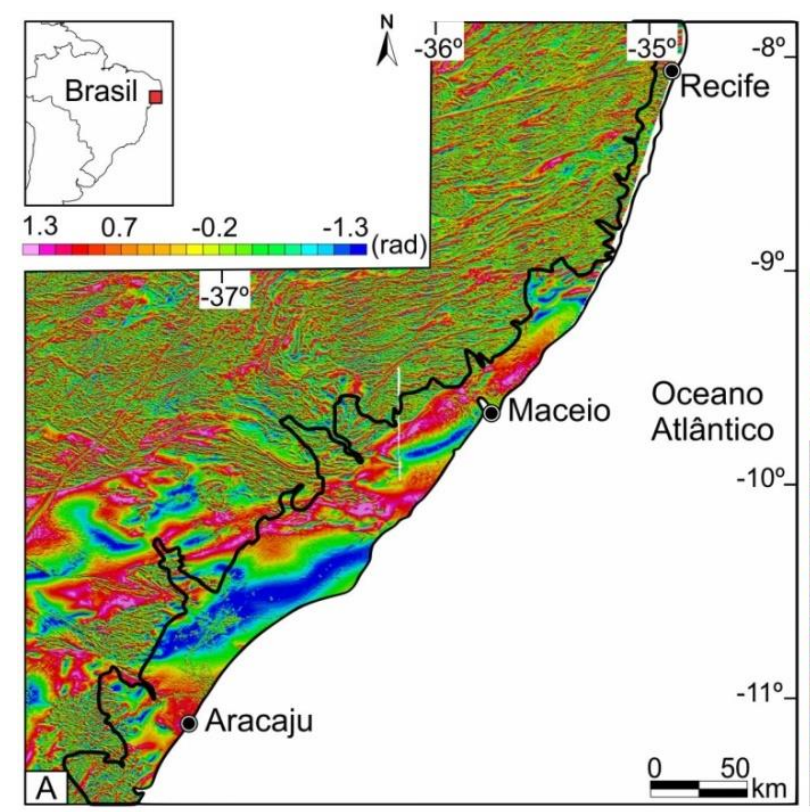

Figura 2 - (A) Mapa da Inclinação do Sinal Analítico (B) com as zonas de cisalhamento compiladas de mapas geológicos.

A ZCBM também foi reativada como falha de borda da bacia Sergipe-Alagoas. Os lineamentos magnéticos de direção E-W e NW-SE, referentes a esta zona, continuam na área da bacia com uma inflexão para NE (Figura 3C). Este padrão ocorre desde o Alto de Sinimbu (A na Figura $3 \mathrm{C}$ ) até o Alto de Maragogi (B na Figura $3 \mathrm{C}$ ), onde a anomalia magnética passa a corresponder à porção norte da falha Tabuleiro dos Martins (C na Figura 3C). O Alto de Maragogi também é afetado por ramificações da ZCJ, onde lineamentos magnéticos de direção NE continuam na bacia na altura desta feição estrutural.

Outro padrão de reativação rúptil de estruturas do embasamento na Bacia Sergipe-Alagoas é registrado através de zonas de cisalhamento com direção NW-SE. Por exemplo, os lineamentos magnéticos referentes à $\mathrm{ZCl}$ continuam na porção da bacia com direção noroeste (A na Figura 3A). Esta continuidade representa a Falha de Vaza-Barris, que é marcada pelo vale de rio homônimo no MDT (Figura 3B). Estruturas com esta direção também são responsáveis pelo controle

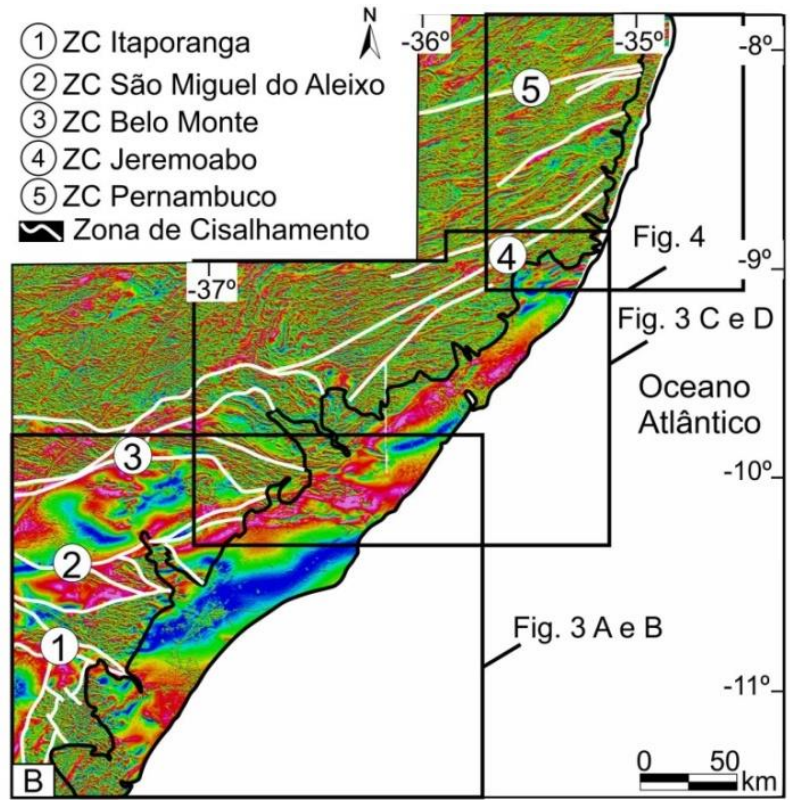

$\mathrm{Na}$ Bacia Sergipe-Alagoas, a evidência mais clara de reativação rúptil de estruturas dúcteis do embasamento é observada nas zonas de cisalhamento SMA e BM. Nós verificamos que os lineamentos magnéticos relativos à ZCSMA possuem continuidade na bacia representada pelos altos de Japoatã e Penedo, respectivamente, B e C na Figura $3 A$. Esta continuidade também é observada através do Modelo Digital do Terreno (MDT), inclusive na borda norte da planície costeira do delta do rio São Francisco (Figura 3B). Esta zona foi reativada como falha de borda da bacia no trecho que corresponde ao Domo de Igreja Nova até 0 Alto de Palmeira Alta, respectivamente, $D$ e $E$ na Figura $3 A$. Nesta última localidade, próximo ao encontro com a ZCBM, a foliação milonítica marcada pelas anomalias magnéticas tendem a mudar da direção NE-SW para E-W.

(2) ZC São Miguel do Aleixo

(3) ZC Belo Monte

4) ZC Jeremoabo

5) ZC Pernambuco

topográfico na bacia Sergipe-Alagoas durante o Cenozoico. Por exemplo, ramificações da ZCSMA, com direção NW (2A na Figura 3B), controlam uma elevação composta por unidades Miocênicas próximo ao vale do Rio Japaratuba. Este vale representa a continuidade de uma destas ramificações na Bacia Sergipe-Alagoas (Figura 3B).

Algo similar ocorre na ZCBM e ao norte desta, onde observamos vários altos e baixos topográficos com direção NW-SE. Os cursos de drenagens próximos às duas ramificações desta zona (3A na Figura 3D) coincidem com a direção das zonas de cisalhamento e falhas do rifte. Por exemplo, na área de bacia, os rios Coruripe, Jequiá e São Miguel representam a continuidade destas ramificações (Figura 3D). Nós interpretamos que, nessa localidade, esses baixos e altos topográficos representam reativações rúpteis destas zonas e de falhas normais do Cretáceo (No caso, a Falha de Coruripe - D na Figura 3D) ocorridas desde 0 Cretáceo até o Quaternário. 

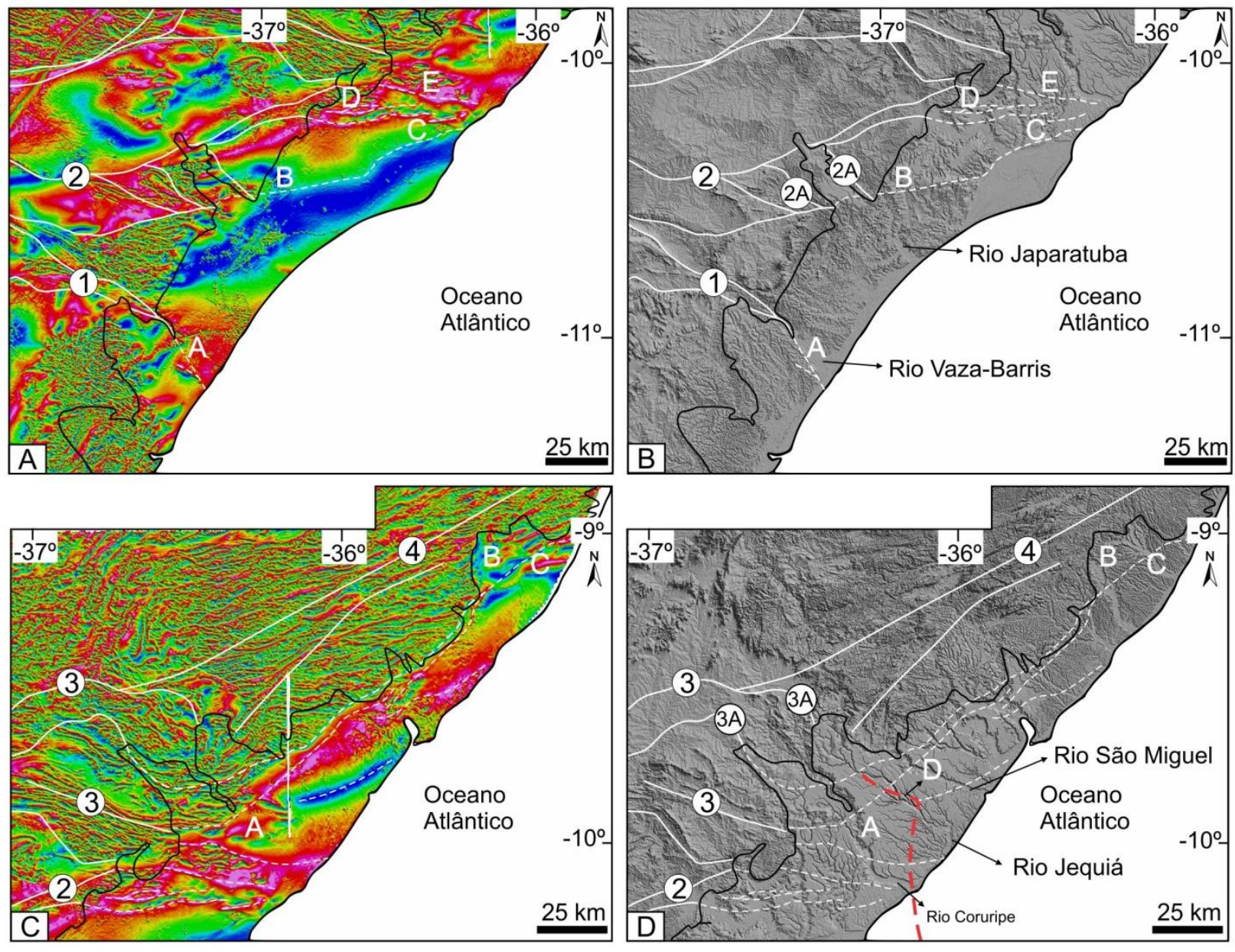

\begin{tabular}{llll}
1.3 & 0.7 & -0.2 & -1.3 \\
\hline
\end{tabular}

Figura 3 - (A) e (C) Mapas da Inclinação do Sinal Analítico e (B) e (D) Modelo Digital do Terreno (Filtro Hillshade 45) com as principais zonas de cisalhamento compiladas e alguns lineamentos magnéticos interpretados na porção da Bacia Sergipe-Alagoas. Linha em preto representa o limite do embasamento com a bacia.

$\mathrm{Na}$ Bacia Pernambuco, a evidência mais clara de reativação rúptil de estruturas dúcteis do embasamento é observada na ZCPE, ZCJ e algumas indiscriminadas. Nós observamos que os lineamentos magnéticos relativos à ZCPE e zonas de cisalhamento indiscriminadas, localizadas ao sul desta, possuem continuidade na bacia (A na Figura 4). O mesmo ocorre com a ZCJ e uma indiscriminada, onde os lineamentos magnéticos referentes a estas zonas possuem continuidade na área bacia. Inclusive, a continuidade desta zona indiscriminada é representada na bacia pelo vale do Rio Pirapama (B na Figura 4B).

Nós também verificamos que algumas falhas representam a continuidade da ZCJ. Por exemplo, a falha do Rio dos Passos (D na Figura 4A) e falhas de borda da bacia Pernambuco (C na Figura $4 \mathrm{~A}$ ) são continuidades da ZCJ e sua ramificação, respectivamente. Inclusive, a Falha do Rio dos Passos é bem visualizada no MDT através de um alto topográfico a noroeste do rio homônimo ( $D$ na Figura 4B). Assim como esta, a ramificação principal da ZCJ é bem marcada no MDT por um alto topográfico (C na Figura 4B).

\section{Discussão e Conclusões}

Nós identificamos, através de mapas aeromagnéticos, a continuidade de zonas de cisalhamento que se encontram no embasamento das bacias Sergipe-Alagoas e Pernambuco e correlacionamos a continuidade destes lineamentos com as falhas mapeadas por trabalhos prévios. Por exemplo, Falkenhein et al. (1986), observaram que a Falha de Vaza-Barris representa a continuidade da $\mathrm{ZCl}$ na bacia Sergipe-Alagoas. Nós corroboramos esta afirmação e verificamos que isto é observado através das anomalias magnéticas e no MDT. Este sistema de falhas marca o limite entre o Cráton São Francisco e a Bacia Sergipe-Alagoas (Falkenhein et al., 1986). Além disso, nós também observamos que, por vezes, as reativações destas zonas de cisalhamento influenciam no relevo. Como é o caso da Planície Costeira do São Francisco, onde Lima et al. (2014), descrevem esta feição como o produto de reativação de 
falhas normais do Cretáceo durante o Quaternário. Nós interpretamos que esta feição representa, através do Alto de Penedo, uma reativação da ZCSMA, a qual é reativada desde o Cretáceo. As reativações da ZCSMA, marcadas pelos altos de Japoatã Penedo ( $B$ e $C$ na Figura $3 \mathrm{~A}$ ) representam o limite entre as sub-bacias
Sergipe e Alagoas (Feijó, 1994). Nós verificamos que essas reativações controlam as falhas de borda, principalmente, na bacia Sergipe-Alagoas, onde a continuidade da ZCBM é representada na bacia pelos altos de Sinimbu e Maragogi. O Alto de Maragogi também é afetado por ramificações da ZCJ.
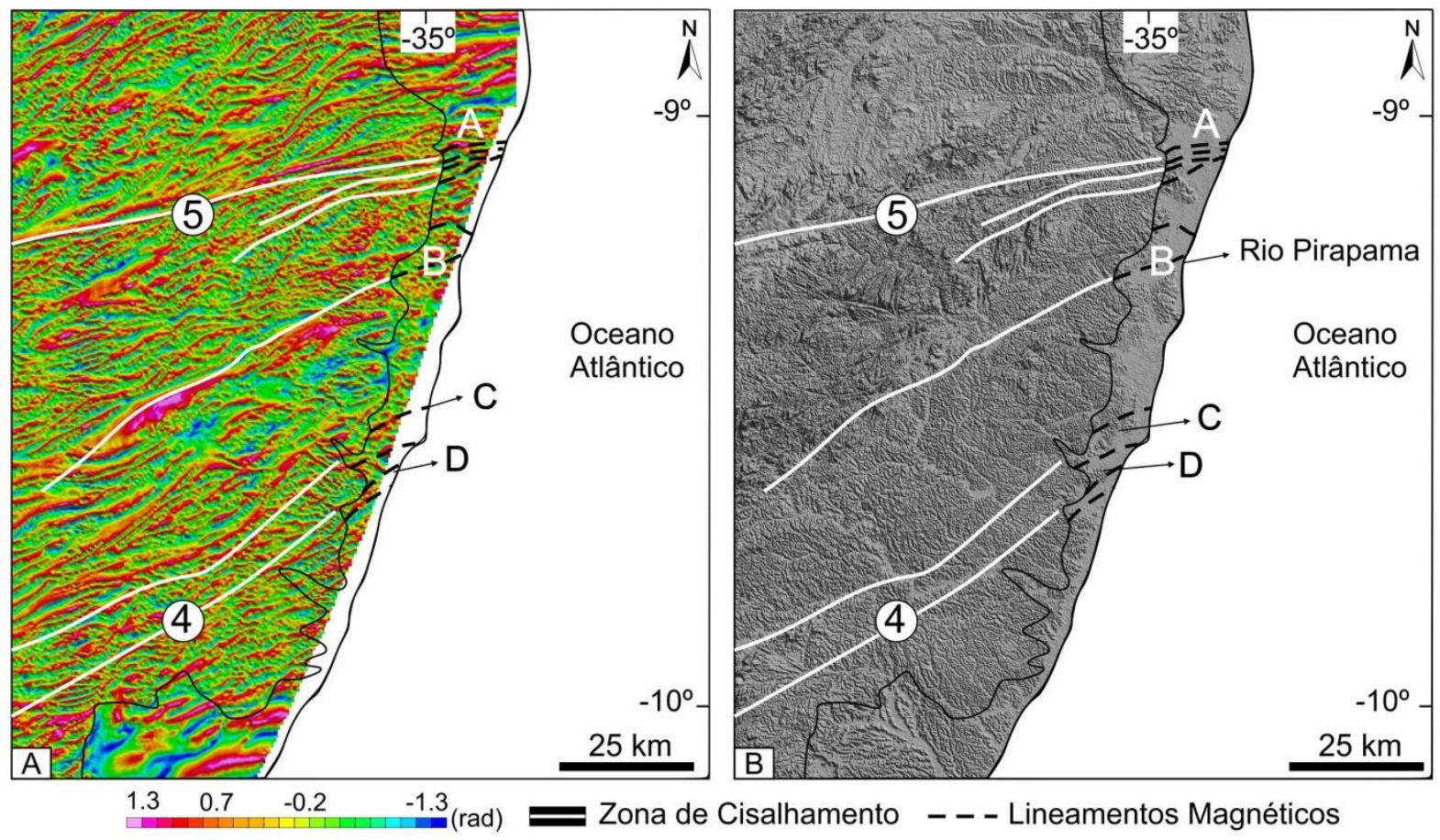

Figura 4- (A) Mapas da ISA e (B) MDT (Filtro Hillshade 315) com as principais zonas de cisalhamento compiladas e alguns lineamentos magnéticos interpretados na porção da Bacia Pernambuco. Linha em preto representa o limite do embasamento com a bacia.

Reativações de zonas de cisalhamento também foram observadas na Bacia Pernambuco. Nós observamos que a ZCJ possui continuidade na bacia representada por falhas normais da fase rifte. Alguns autores, tal como Almeida et al. (2005), mostraram que estas zonas de cisalhamento afetam até unidades do Paleogeno. Contudo, nós concluímos, com base nos dados morfoestruturais, que as zonas de cisalhamento também foram reativadas durante o Cenozoico na Bacia Pernambuco, influenciando na formação de altos e baixos topográficos, como os vales dos rios Pirapama e dos Passos.

Por fim, nós interpretamos que as zonas de cisalhamento foram reativadas, principalmente, no Cretáceo, durante a abertura do Atlântico Sul. Entretanto, assim como Lima et al. (2014) e Lima et al. (2016), nós observamos que tanto estas zonas quanto as falhas normais da fase rifte foram reativadas durante o Cenozoico afetando rochas da Formação Barreiras e os Depósitos Pós-Barreiras.

\section{Agradecimentos}

Nós agradecemos ao Serviço Geológico do Brasil (CPRM) pelo fornecimento dos Projetos Aerogeofísicos. DLV agradece ao CNPq por sua bolsa de doutorado. FHRB e DLC agradecem ao CNPq por suas bolsas de pesquisador.

\section{Referências}

Almeida, C.B. de, Rabêlo Cruz, L., Jardim de Sá, E.F., Vasconcelos, P.M. de P., Medeiros, W.E. de, 2005. Tectônica e relações estratigráficas na Sub-bacia de Pernambuco, NE do Brasil: contribuição ao conhecimento do Rifte Sul-Atlântico. Boletim de Geociências da Petrobras, 13: 167-180.

Angelim, L.A.A., Wanderley, A.A., 2004. Folha SC.25Recife. In: Schobbenhaus, C., Gonçalves, J.H., Santos, J.O.S., Abram, M.B., Leão neto, R., Matos, G.M.M., Vidotti, R.M., Ramos, M.A.B., De Jesus, J.D.A. (eds), Carta Geológica do Brasil ao Milionésimo, Sistema de Informações Geográficas. Programa Geologia do Brasil. CPRM, Brasília. CD-ROM.

Angelim, L.A.A., Camozzato, E., Wanderley, A.A., 2004a. Folha SC.25-Natal. In: Schobbenhaus, C., Gonçalves, J.H., Santos, J.O.S., Abram, M.B., Leão neto, R., Matos, G.M.M., Vidotti, R.M., Ramos, M.A.B., De Jesus, J.D.A. (eds), Carta Geológica do Brasil ao Milionésimo, Sistema de Informações Geográficas. Programa Geologia do Brasil. CPRM, Brasília. CD-ROM.

Barbosa, J.A., Maia, M.F., Magalhães, J.R., Correia, O., 2014. Seismic Stratigraphy of the Onshore Portion of 
Pernambuco Basin: Evidence of Break-Up during Middle Albian for the South Atlantic Rift in Northeast Brazil. In: AAPG Annual Meeting and Exhibition, 2014, Houston.

Basile, C., Maillard, A., Patriat, M., Gaullier, V., Loncke, L., Roest, W., Mercier de Lépinay, M., Pattier, F., 2013. Structure and evolution of the Demerara Plateau, offshore French Guiana: Rifting, tectonic inversion and post-rift tilting at transform-divergent margins intersection. Tectonophysics 591: 16-29.

Bezerra, F.H.R., do Nascimento, A.F., Ferreira, J.M., Nogueira, F.C.C., Fuck, R.A., Neves, B.B.B., Sousa, M.O.L., 2011. Review of active faults in the Borborema Province, Intraplate South America Integration of seismological and paleoseismological data. Tectonophysics 510: 269-290.

Bezerra, F.H.R., Rossetti, D.F., Oliveira, R.G., Medeiros, W.E., Brito Neves, B.B., Balsamo, F., Nogueira, F.C.C., Dantas, E.L., Andrades Filho, C., Góes, A.M., 2014. Neotectonic reactivation of shear zones and implications for faulting style and geometry in the continental margin of NE Brazil. Tectonophysics 614: 78-90.

Brito Neves, B.B., Fuck, R.A., Pimentel, M.M., 2014. The Brasiliano collage in South America: a review. Brazilian Journal of Geology 44: 493-518.

D'el-Rey Silva, L.J.H., 1999. Basin infilling in the southern-central part of the Sergipano Belt (NE Brazil) and implications for the evolution of PanAfrican/Brasiliano cratons and Neoproterozoic sedimentary cover. Journal of South American Earth

Delgado I.M., Souza J.D., Silva L.C., Silveira Filho N.C., Santos R.A., Pedreira A.J., Guimarães J,T, Angelim L.A.A., Vasconcelos A.M., Gomes I.P., Lacerda Filho J.V., Valente C.R., Perrotta M.P., Heineck C.A., 20032 Geotectônica do Escudo Atlântico. In: L.A. Bizzi, C. Schobbenhaus, R.M. Vidotti, J.H. Gonçalves. eds., 2003. Geologia, tectônica e recursos minerais do Brasil. Brasília, CPRM. 227-334.

Eig, K, Bergh, S.G., 2011. Late Cretaceous-Cenozoic fracturing in Lofoten, North Norway: Tectonic significance, fracture mechanisms and controlling factors. Tectonophysics 499: 190-205.

Falkenhein, F.U.H., 1986. Análise da bacia SergipeAlagoas. PETROBRAS/DEPEX/CENPES. Relatório interno da PETROBRAS, $\mathrm{s} / \mathrm{n}^{\circ}$, 210 pp., 9 volumes com mapas. Aracaju.

Feijó, F.J., 1994. Bacias de Sergipe e Alagoas. Boletim de Geociências da Petrobrás, 8(1): 149-161.

Fyhn, M.B.W., Boldreel, L.O., Nielsen, L.H., 2009. Geological development of the Central and South Vietnamese margin: Implications for the establishment of the South China Sea, Indochinese escape tectonics and Cenozoic volcanism. Tectonophysics 478: 184-214.

Kosin, M., Angelim, L.A.A., Souza, J.D., Guimarães, J.T., Teixeira, L.R., Martins A.A.M., Beno, R.V., Santos, R.A., Vasconcelos, A.M., Neves, J.P., Wanderley, A.A., Carvalho, L.M., Pereira, L.H.M., Gomes, I.P., 2004. Folha
SC.24-Aracaju. In: Schobbenhaus, C., Gonçalves, J.H., Santos, J.O.S., Abram, M.B., Leão neto, R., Matos, G.M.M., Vidotti, R.M., Ramos, M.A.B., De Jesus, J.D.A. (eds), Carta Geológica do Brasil ao Milionésimo, Sistema de Informações Geográficas. Programa Geologia do Brasil. CPRM, Brasília. CD-ROM.

LASA PROSPRECÇÕES S/A., 2008. Projeto Aerogeofísico Borda Leste do Planalto da Borborema. MME/CPRM, Relatório Final, Rio de Janeiro, 401 p.

LASA PROSPRECÇÕES S/A., 2011. Projeto Aerogeofísico Estado de Sergipe. MME/CPRM, Relatório Final, $141 \mathrm{p}$.

Lima, C.C.U., Bezerra, F.H.R., Nogueira, F.C.C., Maia, R.P., Sousa, M.O.L., 2014. Quaternary fault control on the coastal sedimentation and morphology of the São Francisco coastal plain, Brazil. Tectonophysics 633, 98114.

Lima, J.C.F., Bezerra F.H.R., Rossetti, D.F., Barbosa, J.A., Medeiros, W.E., de Castro, D.L., Vasconcelos, D.L., 2016. Neogene-Quaternary fault reactivation influences coastal basin sedimentation and landform in the continental margin of NE Brazil. Quaternary International No prelo.

Lima Filho, M.F., 1998. Análise Estrutural e Estratigráfica da Bacia Pernambuco. Tese de Doutorado. Pósgraduação em Geociências - USP, 139p.

Matos, R.M.D., 1992. The northeastern Brazilian Rift System. Tectonics 11: 766-791.

Mao, K., Xie, X., Xie, Y., 2015. Post-rift tectonic reactivation and its effect on deep-water deposits in the Qiongdongnan Basin, northwestern South China Sea. Marine Geophysical Research 36 (2-3): 227-242.

Microsurvey Aerogeofísica e Consultoria Científica Ltda., 2011. Projeto Aerogeofísico Paulo Afonso-Teotônio Vilela. MME/CPRM, Relatório Final, Rio de Janeiro, 255p.

Salomon, E., Koehn, D., Passchier, C., Hackspacher, P.C., Glasmacher, U.A., 2015. Contrasting stress fields on correlating margins of the South Atlantic. Gondwana Research 28: 1152-1167.

Souza-Lima, W., Andrade, E. de J., Bengtson, P., Galm, P.C., 2002. A Bacia Sergipe-Alagoas: evolução geológica, estratigrafia e conteúdo fóssil. Fundação Paleontológica Phoenix, edição especial, 34p.

Storti, F., Salvini, F., Rossetti, F., Phipps Morgan, J., 2007. Intraplate termination of transform faulting within the Antarctic continent. Earth and Planetary Science Letters 260: 115-126.

Valeriano, M.M., Albuquerque, P.C.G., 2010. Topodata: Processamento dos dados SRTM. Instituto Nacional de Pesquisas Espaciais - INPE, São José dos Campos, 79p. (INPE-16702-RPQ/854).

Zitellini, N., Rovere, M., Terrinha, P., Chierici, F., Matias, L., Team, B., 2004. Neogene Through Quaternary Tectonic Reactivation of SW Iberian Passive Margin. Pure and Applied Geophysics 161 (3): 565-587. 